\title{
Tecnologia de informação para a educação na saúde: duas revisões e uma proposta
}

\author{
Information technology for health education: \\ two revisions and a proposal
}

Maria Tereza Leal Cavalcante ${ }^{1}$

Miguel Murat Vasconcellos ${ }^{1}$

Abstract This paper presents two revisions: one discusses the absorption of information technologies in H ealth and Education; the other presents a revision of technological concepts applicable to professional healthcare education. The objectives are to demonstratethe relevance of including these technologies in large-scale institutional training projects for healthcare practitioners in Brazil's $\mathrm{N}$ ational $\mathrm{H}$ ealth System, presenting material and political devices for teaching-learning processes that foster the sharing of content, the reusability of educational materials and interdisciplinarity. This paper thus illustrates the possibilities of adopting standardsand the development of learning objects as content creation, distribution and management technologies. In the political sphere, institutional leadership and cooperation networks are highlighted as key elements for clustering the efforts of academic institutions, training centers and services for collectively building up a $\mathrm{H}$ ealthcare Education Technology base.

Key words Information technology, Healthcare education, Standards, Learning objects
Resumo N este artigo realizam-se duas revisões: a primeira discute a incorporação de tecnologias deinformação na saúdeena educação. A segunda apresenta uma revisão de conceitos tecnológicos aplicáveis na Educação na Saúde. 0 objetivo é evidenciar a pertinência de incorporação de tecnologias de informação em projetos institucionais de formação de profissionais para o Sistema Ú nico deSaúde brasileiro, em larga escala, eapresentar dispositivos materiais e políticos para processos de ensino-aprendizagem em saúde que favoreçam o compartilhamento de conteúdos, a reutilização de materiais educativos e a interdisciplinaridade. Como resultado, ilustram-se possibilidades de adoção de padrões e do desenvolvimento de objetos de aprendizagem, tecnologias apli cáveis para a criação, distribuição e gestão de conteúdos. $\mathrm{N}$ a dimensão política, destacam-se liderança institucional e redes de cooperação como os elementos estruturantes para a articulação de esforços deinstituições acadêmicas, centros deformação e serviços para a criação coletiva de uma base tecnológica para a Educação na Saúde.

Palavras-chave Tecnologia de informação, Educação na saúde, Padrões, O bjetos de aprendizagem

Pública, Fundação Oswaldo

Cruz. Rua Leopoldo

Bulhões 1480/727,

M anguinhos. 21041-210

Rio deJaneiro RJ.

maria.tereza@ensp.fiocruz.br 
Não alimento nenhuma ilusão quanto a um pretenso domínio possível do progresso técnico, não se trata tanto de dominar ou prever com exatidão, mas sim de assumir coletivamente um certo número de escolhas. PierreLevy

\section{I ntrodução}

Vivenciam-se hoje, no Sistema Ú nico de Saúde (SUS), iniciativas voltadas para a Educação na Saúde, que colocam na agenda dos centros de formação e das instituições acadêmicas questões como a reorientação do ensino, a reorganização curricular, a revisão de modalidades de oferta de cursos, de práticas pedagógicas e de conteúdos.

Podem ser de pronto enumeradas iniciativas em curso ou em proposição, nos termos definidos pelo M inistério da Saúde e pela Secretaria de Gestão do Trabalho eda Educação na Saúde(SGTES) ${ }^{1}$, a saber: a Política de Educação Permanentee a Formação deFormadores e deFormuladores de Políticas, que visam a superação das concepções tradicionais de educação e que incorporam como estratégia modalidades de educação a distância² ea RededeEnsino para a Gestão Estratégica do SUS, que propõe estabelecer parcerias e apoiar os processos formativos das diversas Escolas de Saúde Pública e Instituições Públicas de Ensino Superior.

Soma-se a estas iniciativas governamentais a proposição pelo Conselho Nacional de Saúde (CNS) da realização, em 2006, da 3a Conferência N acional de Gestão do Trabalho e da Educação naSaúde, com o objetivo de estabelecer diretrizes para a implementação da Política Nacional de Gestão do Trabalho e da Educação na Saúde (PNGTES). No documento ${ }^{3}$ que subsidiou 0 processo preparativo para Conferência, estão propostas a educação permanente em saúde e a reorientação do ensino em saúde pelo enfoque na integralidade e humanização em saúde. I dentifica-se a necessidade de descentralizar e disseminar capacidade pedagógica no interior do setor saúde, fazendo do SU S uma rede-escola3.

A agenda da Conferência e a forte demanda governamental, expressa nos programas mencionados, colocam para os centros de formação em saúde questões na relação entre oferta e demanda de cursos. Se, por um lado, as instituições formadoras têm o desafio de repensar a estruturação de seus conteúdos, seus processos de planejamento e gestão, por outro, devem fortalecer sua capacidade crítica frente à recomenda- ção de proceder a uma reorientação do ensino.

O campo educacional, como estudado por Struchiner ${ }^{4}$, vive um processo de reformulação favorecido pela incorporação de tecnologias de informação, como ensino à distância e metodologias de educação continuada, em que se destacam a descentralização e a individualização do processo ensino-aprendizagem. Por outro lado, Struchiner ${ }^{4}$ destaca que, apesar da formação em recursos humanos em saúde ser estratégica desde os anos 80, a formação continuada, do ponto de vista pedagógico, tem utilizado métodos de ensino reprodutivistas que conduzem à passividade e à superficialidade.

A exigência de mudanças nos conteúdos e projetos de formação profissional tampouco é exclusiva da Saúde. Santos ${ }^{5}$ identifica nas Ciências Sociais a necessidade de repensar eatualizar os seus conteúdos frente à transição de paradigmas científicos em que se exigeo desenvolvimento de um pensamento complexo e relacional e, para tanto, discute a formação dos cientistas sociais em metodologias computacionais.

Esse cenário desafia às instituições de ensino de saúde a:

a) posicionar-se criticamentefrente à agenda da educação na saúde

b) proceder à inovação dos processos de ensino-aprendizagem

c) aumentar a produção de conhecimento sobre o uso de tecnologias de informação em saúdena pesquisa, no ensino-aprendizagem e na gestão do sistema de saúde.

A adoção de metodologias de compartilhamento de conteúdos em redes de escolas pode ser um elemento estruturante da resposta a estes desafios. N este artigo, ilustram-se possibilidades tecnológicas, como a adoção de padrões e do desenvolvimento de objetos de aprendizagem em instituições de ensino em Saúde, ao mesmo tempo em quese revisam recursos políticos para uma resposta coletiva, em rede, por parte de instituições acadêmicas, centros de formação e serviços.

Duas revisões de literatura são realizadas: a primeira discute a incorporação de tecnologias de informação na saúde e na educação ao longo das décadas de 90 até atualidade. São evidenciadas convergências e lacunas nessa literatura earticula-se as produções de I nformações em Saúde com as deEducação na Saúdeno que diz respeito à incorporação de tecnologias de informação.

A segunda revisão apresenta conceitos tecnológicos aplicáveis na Educação na Saúde. Em especial, são apresentados os conceitos de objetos de aprendizagem, as iniciativas de padronização 
de metadados e os consórcios interinstitucionais para compartilhamento de conteúdo.

Por fim, a conjugação das lacunas e das potencialidades das duas revisões, sem que se estabeleça protagonismo de uma ou de outra, permite desenhar uma proposta na qual são evidenciados os dispositivos políticos e materiais para a estruturação de uma base tecnológica comum para a Educação na Saúde no SUS.

\section{Tecnologias deinformação na saúde e na educação: uma trajetória em revisão}

Na década de 90, Lévy ${ }^{6}$ circunscreve as tecnologias de informação a um campo político aberto e conflituoso. A significação eo papel de uma configuração técnica em um dado momento não podem ser separados do projeto que move esta configuração, nem mesmo dos projetos rivais que a disputam. Indaga em que medida certos projetos e atores escapam de uma visão imediatista, racionalizadora e utilitária destas tecnologias, em que se privilegie outras bel ezas que não as do espetáculo e do lucro.

Ilustra com a informática escolar na França nos anos 80 onde, apesar dos vultuosos recursos, das escolas equipadas e dos professores formados, os programas fracassaram. As resistências sociais são explicadas por Levy": ao procurar a imagem da modernização, o governo não obteve nada além de imagens, não houve a condução de um projeto político em que seanalisassem as transformações em andamento eos novos modos de constituição e transmi ssão do saber.

$\mathrm{N}$ a mesma década em que se destaca a produção de Levy, M oraes ${ }^{7}$ discute na Saúde a difusão de certa euforia com a chamada Era da Informação e trata a informação como espaço estratégico, a partir das distintas formas que assumem as relações entre o Estado e a sociedade. Seu trabalho analisa a evolução dos Sistemas de Informação em Saúdeno País como expressão da fragmentação institucional existente. Identificaredundância de esforços, defasagens de tecnologia e gestão inadequada. Em 1998, apóia-se nos estudos de M ichel Foucault e conclui pela necessidade de ampliação da capacidade argumentativa dos sujeitos informacionais para um processo democrático emancipador.

Também em 1998, Iturri ${ }^{9}$ analisa aspectos sociais da incorporação de tecnologias de informação e comunicação em instituições de ensino e pesquisa de saúde pública. Enumera pontos críticos: dificuldades de aquisição e manutenção de base tecnológica, resistência de professores e pesquisadores para aprender e desenvolver tecnologias, percepção que essa tecnologia requer esforço etempo desmedidos em comparação aos benefícios, percepção de que a maior parte das aplicações estão desenhadas para atender a requisitos do mercado - enão a necessidades específicas do ensino epesquisa - e por fim, dificuldades para constituir processos de compartilhamento de informações entre colegas de instituição e com pesquisadores de outras instituições.

Por outro lado, em 2000, do ponto de vista do apoio à decisão clínica e gerencial, Leão ${ }^{10}$ re comenda integrar a informação com o estabelecimento de comunicação entre sistemas de informações independentes e heterogêneos. Em 2002, Vasconcellos ${ }^{11}$ evidencia a defasagem existente entreo avanço do conhecimento no campo das tecnologias de informação e a incorporação destas tecnologias ao processo de gestão em saúde no Brasil.

Com a intensificação do uso de tecnologias de informação na Educação à Distância (EAD) em Saúde, elementos positivos deste processo são evidenciados na produção de M isoczky ${ }^{12}$, Chris$\operatorname{tante}^{13}$ e Costa $^{14}$. M isoczky ${ }^{12}$ defende uma proposta educativa voltada à mudança eà pluralidade - e não ao conformismo ea submissão - e percebe a EAD como uma estratégia para ( $r$ re) significação do processo educativo. Salienta que as possibilidades de transgressão provêm da ambiência humana implicada no processo formativo. Outros estudos ${ }^{15,16}$ centram a atenção nas plataformas, ambientes, ferramentas, interações e estratégias pedagógicas, com relatos de casos.

Essa literatura ${ }^{12,13,14,15,16}$, embora dialogue com um ideal político transformador no uso de tecnologias na educação, o faz do ponto de vista pedagógico enão institucional. Oscasos eas análises ilustram mediações entre tecnologias e concepções pedagógicas na estruturação e na oferta de determinados programas e cursos. Há lacunas em relação à dimensão tecnológica material capaz de suportar projetos políticos institucionais de maior escala para responder as necessidades de formação de profissionais para o SUS.

$\mathrm{Na}$ Educação, desde 2001, Belloni ${ }^{17}$ expressa preocupação com a incorporação acelerada eacrítica de tecnologias em programas educacionais degoverno. Recorreà Debord, $\mathrm{H}$ abbermas, $\mathrm{M}$ arcuse e $M$ attelart na composição de suas críticas ao modelo brasileiro de incorporação de tecnologia à educação à distância. Alerta que no contexto atual do capitalismo, o campo educacional aparece como uma nova fatia de mercado promis- 
sora ${ }^{17}$. Discute a fragilização do papel da escola como agência de socialização e analisa alguns programas de educação à distância brasileiros que, mesmo fundamentados em teorias psicopedagógicas inovadoras (como construtivismo, sociointeracionismo e interculturalismo), não conseguem romper tendências enraizadas nainstituição escolar.

(...) a questão fundamental não estaria tanto na modalidade do ensino oferecido - se em presença ou à distância, mas, sobretudo na capacidadede os sistemas ensinantesinovarem quanto aos conteúdos e às metodologias de ensino, de inventarem novas soluções para os problemas antigos e também para aqueles problemas novíssimos gerados pelo avanço técnico nos processos de informação e comunicação, especialmente aqueles relacionados com as novas formas de aprender ${ }^{17}$.

Convém reproduzir as ressalvas realizadas em 2005 por Jiménez ${ }^{18}$ às tecnologias de informação na educação, cuja incorporação considera não representar uma questão metodológica presumidamenteneutra, concernente ao aperfeiçoamento dos procedimentos destinados à consecução de objetivos pedagógicos de currículo. Para Jiménez ${ }^{18}$, a questão central, base de toda discussão sobre a educação, é o papel primordial que os dispositivos pedagógicos representam nos processos de produção, reprodução e transformação da cultura dominante e das relações de poder vinculadas a ela. 0 comando pedagógico - próprio do que Jiménez ${ }^{18}$ denomina Capitalismo (Disciplinário) de Redes - responde ao desenvolvimento de lógicas de dominação complexas e a estratégias de poder que cristalizam, concretizam e se instalam em uma nova Escola cada vez mais permeada pelo pragmatismo capitalista. Trata-se da Escola do Espetáculo e H egemonia Tecnocapitalista. As principais referências de J iméne $z^{18}$ para esta leitura são Gramsci, Baudrillard, Foucault, Bernstein e Debord.

Aqui retornamos quase uma década parailustrar que Nunes ${ }^{19}$, em 1998, ao recuperar os marcos da formação de sanitaristas no Brasil nas décadas de 70 e80, entende o fenômeno educativo como "objetivação da produção intelectual dos grupos que conformam as instituições pedagógicas em um determinado momento, portanto, fruto de relações sociais".

Nessas instituições se realizam as idéias pedagógicas, em torno das quais convivem, dialeticamente, o caráter progressista com 0 conservador, 0 novo e o velho, o dominante e o dominado. As instituições pedagógicas são espaços em que as si- tuações contradi tórias são consider adas germes dos processos de criação $0^{19}$.

Contemporânea aos estudos de Moraes ${ }^{8}$, Nunes ${ }^{19}$ baseia-se no entendimento de educação de Cury ${ }^{20}$, em que esta assume um caráter mediador propositivo em que os homens são agentes históricos e não meros produtos sociais. Nunes ${ }^{19}$ refere-se a Cury ${ }^{20}$ para ressaltar que a educação é produto humano e conservará o caráter dialético dosfenômenos existentes na estrutura social eque, se os modos de produção são mediados entre os homens, os homens mediados podem se converter em mediadores entre a estrutura econômica e um novo homem.

Frente ao exposto, como desenhar uma base tecnológica inovadora para os processos de ensino-aprendizagem em saúde que favoreça o compartilhamento de conteúdos, a reutilização de materiais educativos, a interdisciplinaridadee a incorporação de diferentes discursos em Saúde? Quais tecnologias constituem uma possível base material capaz de suportar projetos políticos institucionais que respondam às necessidades de formação de profissionais para o SUS em larga escala?

Dentre os elementos fundamentais para responder estas questões estão os objetos de aprendizagem e os padrões de metadados, apresentados e discutidos a seguir.

\section{Orientação a objetos}

Do universo das tecnologias de informação e comunicação emergem os $\mathrm{O}$ bjetos de Aprendizagem inspirados pelos modelos de Orientação a Objetos da Engenharia de Software.

A Orientação a Objetos, conforme apresentada por Yourdon ${ }^{21}$, nasceu da engenharia de software baseada em modelos que, por sua vez, regese pelo princípio da separação das preocupações, expresso pela construção de um modelo de análise separado de um modelo de projeto. Os modelos de análise capturam requisitos essenciais deum domínio de problema e descrevem o comportamento desejado, independentede qualquer abordagem de implementação ou tecnologia específica. Os modelos de projeto são construídos por quem possui extenso conhecimento do ambiente de implementação emostram em detalhes como um sistema específico seráconstruído ( plataforma, rede, sistema operacional, banco de dados, interface com o usuário ${ }^{21}$.

0 modelo de análise orientada a objetos é um esforço de abstração de um determinado 
problema ou processo em que se definem quais são as coisas do mundo real a modelar e se estabelecem blocos de construção básicos a partir do qual o sistema será construído, estruturado em componentes de um domínio de problema ${ }^{22}$.

Esses componentes são os objetos. Cada objeto éuma entidadeindependente, quesabe coisas (detém informações); realiza trabalhos (tem funcionalidades) e colabora com outros objetos para executar funções finais de um sistema. A vantagem básica da orientação a objetos é permitir a reusabilidade em larga escala destes diversos componentes, ao tornar possível reutilizar, em sistemas diferentes, estas mesmas unidades já modeladas ${ }^{21,22}$.

\section{O bjetos de aprendizagem: revisão conceitual}

Deforma similar, o conceito de O bjetos deAprendizagem aplica-se a materiais educacionais projetados e construídos em pequenos conjuntos ou blocos com os quais se estrutura o conteúdo de aprendizagem.

Os objetos de aprendizagem são comumente definidos como recursos digitais com características de acessibilidade ( possuem uma identificação padronizada que garante a sua recuperação); reusabilidade (uma vez recuperados são utilizados para compor " $n$ " unidades de apren dizagem), interoperabilidade (tem capacidade de comunicar e funcionar em diversos sistemas). Os objetos são armazenados em repositórios de aprendizagem - bancos de dados que podem ser locais (em uma só instituição) ou distribuídos (por exemplo, em um consórcio de instituições) ${ }^{23}$.

O termo foi popularizado em 1994 nos Estados Unidos, por inspiração de Hodgins, que entendeu, ao ver um de seus filhos brincando com blocos Lego, ser necessário desenvolver peças de aprendizado interoperáveis do tipo plug and play. Nomeou no Computer Education $M$ anagers Association - CEdM A um grupo de trabalho chamado Learning Architecture \& Learning Objects Task Force, com o propósito de desenvolver metodologias para que conteúdos novos e preexistentes pudessem funcionar como objetos de aprendizagem independentes, reunidos em quaisquer combinações para atender necessidades de aprendizado individuais ${ }^{24}$.

Embora inúmeras organizações internacionais dediquem-se a promover a adoção de padrões interoperáveis e suas recomendações sejam defato mundialmente utilizadas em programas e serviços de aprendizagem, na última déca- da os Objetos de Aprendizagem colecionaram definições por vezes imprecisas, tratadas a seguir por Polsani ${ }^{25}$ :

. Um objeto de aprendizagem é definido como uma entidade digital ou não digital, que pode ser usada para aprendizado, educação ou treinamento. (Learning O bject M etadata LOM /IEEE - Learning Technology Standards Committee) ${ }^{26}$

. U m objeto deaprendizagem équalquer recurso digital quepossa ser reusado para dar suporteao aprendizado. Inclui qualquer coisa que possa ser oferecida pela rede ou por demanda, em qualquer escala - fotos, vídeos, áudios, extratos de textos, animações, imagens, pequenas aplicações da web ou mesmo páginas inteiras desde que forneçam experiências completas tais como um evento instrucional completo27.

. U m objeto de aprendizagem édefinido como a menor estrutura independente que contenha um objetivo, uma atividade deaprendizado euma avaliação ${ }^{28}$.

No Brasil, são exemplos de literatura de O bjetos de Aprendizagem usando os conceitos de Wiley e do comitê do IEEE os trabalhos de Tarouco $^{23}$ e Warpechowski ${ }^{29}$, ambos da Universidade Federal do Rio Grande do Sul.

No entanto, para garantir a reusabilidade de objetos de aprendizagem, é necessário que estes sejam descritos em um formato comum, para que possam ser facilmente pesquisados, recuperados e reutilizados para a construção de cursos, treinamentos e novos conteúdos. Esta descrição de formatos comuns é possível pela padronização dos chamados metadados. M etadados são descrições dos dados existentes em um sistema com o objetivo de garantir armazenamento, recuperação e manipulação eficientes ${ }^{30}$. Exemplos de metadados desen volvidos e aplicados na Saúde são os descritores em Ciências da Saúde da Biblioteca Virtual de Saúde da Bireme (Centro Latino-Americano e do Caribe de Informações em Ciências da Saúde) para uso na indexação de artigos derevistas científicas, livros, anais decongressos, relatórios técnicos, e outros tipos de materiais, em bases de dados como LILACS e M EDLIN E ${ }^{31}$. N esse caso, trata-se de um conjunto de termos com o propósito de organizar e recuperar material bibliográfico. No caso dos objetos de aprendizagem, os metadados recuperam objetos que podem estar armazenados na forma de vídeos, imagens, textos, áudios e que detêm informações (conteúdo), possuem funcionalidades (realizam tarefas) e relacionam-se com outros objetos para compor unidades maiores ou cursos. 
Vale destacar que, de acordo com al SO ${ }^{32}$ (International Organization for Standardization), um padrão é um documento aprovado por um organismo reconhecido que provê, pelo uso comum e repetitivo, regras, diretrizes ou características de produtos, processos ou serviços cuja obediência não é obrigatória.

Os padrões ISO ${ }^{32}$ e de outros grandes consórcios internacionais, como o IEEE ${ }^{26}$, são frutos de acordos e consen sos voluntários entre delegações nacionais e outras partes interessadas, como fornecedores, usuários, entidades de regulação governamental e consumidores. Por consenso, acordam-se especificações e critérios que devem ser consistentemente aplicados na produção ou na provisão dos serviços em questão ${ }^{32}$. Os padrões internacionais garantem um modelo de referência e uma linguagem tecnológica comum para o fornecimento econsumo de produtos e serviços, o que facilita a transferência de tecnologia $a^{10,11,26,32}$. São exemplos de padrões adotados na Saúde a Classificação Internacional de Doenças - $\mathrm{CID}^{10}$ e os padrões do Health Level Seven $(H L 7)^{10}$, comunidade internacional de expertos em Saúde e Ciência da Informação que colaboram para criar padrões para a troca, gerenciamento e integração de informações el etrônicas de saúde ${ }^{10,33}$.

$\mathrm{Na}$ última década, diversas organizações internacionais disputam o protagonismo na padronização e na oferta de tecnologias para o desenvolvimento de $\mathrm{O}$ bjetos de Aprendizagem. Os padrões desenvolvidos por estas organizações buscam consenso sobre quais são os metadados necessários para descrever um objeto de aprendizagem. U ma vez aceitos, estes padrões permitem a criação, o armazenamento e a recuperação de objetos em diversas plataformas tecnológicas.

É representativa a atuação do Learning Technology Standards Committee (LTSC) do IEEE ${ }^{26}$, cuja missão é desenvolver padrões técnicos para a interoperação decomponentes ede sistemas de educação e treinamento em computadores. 0 desenvolvimento depadrões érealizado por meio do grupo de trabalho Learning Object M etadata (LOM ) por uma combinação de encontros presenciais, teleconferências e trocas em grupos de discussão. O Padrão LOM ${ }^{26}$ especifica a sintaxee a semântica de metadados de objetos de aprendizagem etem como foco o conjunto mínimo de atributos necessários para que possam ser gerenciados, localizados e avaliados.

Outra iniciativa significativa em Objetos de A prendizagem foi realizada pelo $\mathrm{ADL}^{34}$ - Advanced Distributed Learning do Departamento de
Defesa dos Estados U nidos, que desenvolveu um conjunto de modelos de referências para o compartilhamento de conteúdos, conhecido como SCORM ${ }^{34}$ (Sharable Content Object Reference Model).

Os modelos de referência são coleções de especificações epadrões do IEEE Learning Technology Standards Committee (como o padrão LOM ${ }^{26}$, por exemplo), do IMS Global Learning Consortium ${ }^{35}$, daAICC (Aviation Industry Computer-Based Training Committee) ${ }^{36}$ e da Aliança Européia Ariadne ${ }^{37}$. Estes model os garantem que os conteúdos sejam modelados de forma padronizada, viabilizando assim, em larga escala, a sua utilização em quaisquer plataformas educacionais, chamadas pelo SCORM de LM S (Learning $M$ anagement Systems). Uma vez modelados sob os parâmetros do SCORM - conteúdos e sistemas educacionais na WEB - estão aptos a intercambiar objetos e funcionalidades ${ }^{34}$.

Reunindo os padrões LOM ${ }^{26}$ (metadados de objetos de aprendizagem) e H L $7^{10}$ (trocas eletrônicas de informações em saúde) e apoiado nos modelos de referência do SCORM ${ }^{34}$, destaca-se o Consórcio M edBiquitous ${ }^{38}$, fundado pela Johns H opkins M edicinee por sociedades médicas de reconhecida liderança, incluindo organizações internacionais, associações de assistência à saúde, universidades, organizações comerciais e não governamentais. O Consórcio M edbiquitous é um exemplo concreto da conjugação de esforços interinstitucionais para a padronização, o compartilhamento de conteúdos educacionais em saúde. Baseado nas recomendações do IEEE, 0 $M$ edBiquitous desenvolveu o $\mathrm{H}$ ealthcare Learning O bject M etadata ${ }^{39}$, que provê um modo padronizado de descrever atividades e recursos educacionais em saúde.

Com um grupo de trabalho especialmente dedicado a desenvolver padrões para a interoperabilidade, accessibilidadee reusabilidade deconteúdos de saúde em sistemas de aprendizado na Web, o consórcio utiliza-seainda do padrão XM L (eXtended $M$ arkup Language), que torna mais fácil a troca de dados estruturados pela Internet e deWeb Services, aplicações que possuem interface baseadas em XM L e que descrevem uma coleção de operações acessíveis através de rede, independentemente da tecnologia usada na implementação do serviço ${ }^{40}$. Um dos maiores benefícios dessa interface é a abstração dos detalhes de implementação do serviço, permitindo que seja acessado independente da plataforma dehardware ou software na qual foi implementado.

A pesar das propostas de uso generalizado de 
objetos de aprendizagem em todos os campos formativos, há dúvidas sobre as implicações que esses possam ter nos diversos modelos educativos. Em especial, Domingue ${ }^{41}$ critica a sua origem no mei o militar, bem como o vínculo estreito com interesses empresariais de barateamento de custos de produção para um mercado de ensino virtual destinado à comercialização mais do que à formação.

Longe de descartar as possibilidades de compartilhamento de conteúdos, os críticos ${ }^{41}$ ressaltam outras possibilidades, motivados pelo desenvolvimento detecnologias como o EM L (Educational Modelling Language) da Open University de Nederland (OUNL), que permite representações do processo educativo, com explícita menção às distintas alternativas pedagógicas. $\mathrm{Da}$ mesma forma, no campo de metadados, embora haja consenso de uso do XML, outros autores estudados por Domínguez ${ }^{41}$ propõem a utilização deRDF (Resource Description Framework), elevando a idéia de metadados ao âmbito mais avançado conceitualmente de Web Semântica proposto pela $\mathrm{W}^{3} \mathrm{C}^{40}$.

Ressalvas também são realizadas aos repositórios de objetos de aprendizagem. Pedreño ${ }^{42}$ defende que esses não devem configurar-se em um repositório de materiais, mas sim em uma coleção de recursos aos quais os docentes atribuem certa inteligência pedagógica. U ma biblioteca de objetos de aprendizagem, mais do que de materiais, é uma biblioteca de usos docentes.

Algumas instituições acadêmicas brasileiras já estudam, desenvolvem e aplicam vocabulários, padrões e repositórios para objetos de aprendizagem. São exemplos o Laboratório Virtual ${ }^{43}$ da Escola do Futuro da Universi dade deSão PauIo/U SP, a Pontifícia UniversidadeCatólica do Rio de Janeiro ${ }^{44,45,46}$ e a Universidade Federal do Rio Grande do Sul23,29.

No âmbito governamental nacional, pode-se citar a RIVED - Rede Interativa Virtual de Educação ${ }^{47}$, contrapartida do Brasil na Rede Latinoamericana de Portais Educativos - RELPE ${ }^{48}$. O RIVED é um programa da Secretaria de Educação à Distância - SEED do M inistério da Educação, que tem por objetivo a produção de conteúdos pedagógicos digitais na forma de objetos de aprendizagem. Os conteúdos desenvolvidos nos portais dos dezesseis países membros da RELPE são de livre circulação na rede, o que é possível pelo uso do padrão XML.

Por fim, o Campus Virtual da Organização Pan Americana de Saúde ${ }^{49}$ apresenta um modelo estratégico em rede e um repositório de Objetos de Aprendizagem com base no padrão SCORM . U sando a plataforma educativa livre MOODLE, esse Campus V irtual tem a intenção de potencializar a gestão do conhecimento e dos programas de educação permanente para o desenvolvimento dos recursos humanos em saúde pública.

\section{Por uma basetecnológica para a educação na saúde}

Ao realizar uma reflexão sobre o estado da técnica e da política no mundo contemporâneo, M ilton Santos ${ }^{50}$ conclui que vivemos sob um estado de unicidade das técnicas, um mundo que aparentemente caminha para a homogeneização, com vocação para um padrão único em que impera a mundialização da mais-valia. N o entanto, salienta que sob determinadas condições políticas, a materialidade simbolizada pelo computador é capaz de superar o imperativo da tecnologia hegemônica e favorecer novos processos criativos.

0 potencial transgressor deste e de outros discursos ${ }^{6,8,12,17,18}$, que se detém entre o uso hegemônico enão hegemônico das tecnologias, entre a determinação ou a subordinação das tecnologias aos processos sociopolíticos, ganha uma outra dimensão quando se acrescentam as contribuições de Latour ${ }^{51}$.

Ao tentar situar de outra maneira a produção da ciência e da tecnologia, e a eterna relação entre sociedade e natureza, Latour propõe uma dimensão a mais ao eixo sujeito-objeto. Acrescenta um gradiente de estabilização no qual natureza e sociedade transitam e se co-produzem coletivamente. Recorre aos quasi- objetos de Serres para designar o que circula pelo coletivo sendo modelado por sua própria circulação e ao conceito deator rede de Callon, que expressa essa mesma função de dupla construção entre natureza e sociedade.

Estudos que tentam compreender as relações entre tecnologia de informação esociedadea partir dessa co-produção examinam não apenas o sistema tecnológico ou o sistema social, ou mesmo os dois sistemas lado a lado, mas sim investigam o fenômeno que emerge quando os dois interagem. Autores como Hanseth, Aanestad e Berg ${ }^{52}$ discutem a teoria do ator rede de Callon como uma teoria social da tecnologia.

Esses autores discordam das explicações estruturalistas que estabelecem uma distinção entreartefatos tecnológicos (máquinas, programas, técnicas) e tecnologias em prática (estruturas humanas habilitadas no uso cotidiano dos artefa- 
tos). Recorrem à Teoria do Ator Rede porque supõem a existência de uma rede onde todos são chamados atores e não há distinção, a priori, de nenhum tipo de elemento: seres humanos, artefatos tecnológicos, organizações, instituições, dentre outros. Essa teoria supõe que todas as redes são heterogêneas ou sociotécnicas e que, portanto, não há nenhuma rede constituída somente por seres humanos ou por componentes tecnológicos. Isso significa que o "artefato tecnológico" étambém em si uma redequeinclui seres humanos ou organizações, tão heterogênea como a tecnologia em prática.

No caso da constituição de uma base tecnológica para a Educação na Saúde, os conceitos de ator rede e de redes sociotécnicas permitem romper com o dilema entre a valorização excessiva da técnica em detrimento dos processos pedagógicos e políticos e vice-versa. Sem atribuir a priori nenhum protagonismo, o conceito de ator rede não subjuga uma dimensão à outra e permite relações mais si métricas entre sociedadeetecnologia.

É precisamente nesse espaço heterogêneo da rede sociotécnica, no qual se encontram presentes as tecnologias de informação, que se tecem as condições para uma Coletividade Científica Informacional ${ }^{5}$ - lócus de interação entre pesquisadores e de inter-relações sociais envolvidas na produção da ciência e da tecnologia.

No entanto, Nunes ${ }^{19}$ identifica que a rede de relações não se processa espontaneamente e que as redes de cooperação introduzem idéias de monitoramento, aumentando a capacidade de gestão dos seus processos internos, e, portanto, de recriação de suas práticas associativas. 0 desenvolvimento dos projetos colaborativos está associado a uma seqüência de negociações, que começa com a problematização e envolve os ele mentos dos diversos grupos, dando início a uma seqüência de operações de tradução, ao longo das quais os objetos e produtos se redefinem, até constituírem o produto final ${ }^{53}$.

Neste sentido, sugere-se que instituições de ensino em saúde com processos educacionais com base em tecnologias (presenciais ou à distância) discutam no campo institucional epolítico a presença desses recursos tecnológicos em suas redes. Isto porque a constituição de uma base tecnológica para a Educação na Saúde requer uma apropriação coletiva de técnicas em decisões tomadas pelo conjunto de escolas, centros de formação e instituições de ensino.

Trata-se de transcender a usual abordagem instrumental das tecnologias de informação no processo de ensino-aprendizagem (mediações tecnologia-professor-aluno) para os processos institucionais de pesquisa, métodos de investigação, ensino, formação de redes e gestão. Essa perspectiva acrescenta outras possibilidades à forma de pensar a modelagem dos conteúdos educacionais, tradicionalmente limitados a cada escola, disciplina ou curso, segundo linhas programáticas departamentais, expertise de professores e pesquisadores e demandas de programas de governo.

Deve-se considerar o desenvolvimento de conteúdo e de formas de aprendizado de modo dialógico entre escolas, em operações de coordenação de comportamentos com outras e com si mesmas, pelas quais se produz um mundo de ações possívei $5^{54}$. Estas possibilidades estão na base do processo dialógico e consensual do estabelecimento de padrões e das redes em que não se pactuam apenas uma sintaxe e uma semântica para os conteúdos, mas nos quais estão em jogo projetos políticos institucionais. A construção deum padrão para a representação de metadados de objetos de aprendizagem em saúde éum requisito essencial ao compartilhamento de conteúdos entre instituições de ensino e serviços de saúde.

A padronização exige uma formalização voluntária dos saberes institucionais e disciplinares ea modelagem de conteúdos exige rigoroso processo de análise e abstração, com o objetivo de formalizar e tornar consensual quais elementos e requisitos essenciais dos objetos podem ser recombinados entre diversas disciplinas, cursos e programas. Essa técnica favorece a interdisci plinaridade e o enfoque intersubjetivo habermasiano já defendido por Artmann ${ }^{55}$ que, reunindo conceitos de Si ebeneichcler eJapiassu, defineinterdisciplinaridade como a busca da superação das fronteiras disci plinares pelo estabel ecimento de uma linguagem consensualmente construída entre os cientistas.

Do mesmo modo, o estabelecimento de grupos e comitês interinstitucionais de padrões e modelagem de conteúdo, amparados em uma prática argumentativa, fortal ece a capaci dade crítica frente à recomendação de proceder a uma reorientação do ensino. A forte demanda governamental, expressa nos diversos programas mencionados, e a oferta de cursos para a formação de mão-de-obra em programas específicos de governo, colocam para os centros de formação delicadas questões na relação entre oferta e demanda de cursos, em que é preciso ir além da cena das mídias e discutir os processos sociotécnicos implicados nestes programas (as estratégi- 
as de educação à distância, as tecnologias selecionadas, os conteúdos escolhidos, a orientação pedagógica e a gestão).

Também o modelo de separação das preocupações (em análise e em projetos) da orientação a objetos contribui para o estabelecimento de relações mais claras e simétricas entre formuladores de conteúdos de saúde e equipes de implementação de infra-estrutura tecnológica, contribuindo para a superação das fobias tecnológicas por parte dos primeiros ${ }^{9}$ e do monopólio da técnica por parte dos segundos.

Resta ainda uma dimensão a tratar: a da implementação - como desenhar esta base tecnológica para os processos de ensino-aprendizagem em saúde?

Santos Júnior ${ }^{56}$ ilustra que, para 0 amplo e bom uso das tecnologias da informação, é preciso haver orientação, estímulo, vontade política, liderança, comprometimento, compartilhamento devisões, planejamento, capacidade deassimilar inovações e consciência por parte de toda a organização, notadamente da alta administração.

Sobre a vontade política, $M$ atus ${ }^{57}$ afirma que esta se manifesta no desenvolvimento de uma estratégia para construir a viabilidade de um determinado plano. Essa vontade exige que o deve ser predomine sobre o pode ser. A relação desigual dever ser - poder ser encarna a vontade do líder, expressa no esforço extremo para superar as restrições.

Lídere ${ }^{58}$ são capazes de inventar novas práticas fazendo distinções que não estavam presentes antes e seduzem uma comunidade a adotá-las. Suas ações dão forma ao futuro. Transcender o atual modelo de incorporação de tecnologias na educação na saúde, com discussões localizadas em cada escola e aquisição de plataformas heterogêneas, exigelideranças institucionais capazes de fazer distinções e declarar possibilidades futuras, tecendo uma visão crítica e coletiva do uso desses recursos.

Em termos práticos, vontade política, uma liderança institucional e redes de cooperação se traduzem nos elementos estruturantes de uma aposta coletiva de construção de uma base tecnológica para a educação na saúde, quebem pode se iniciar com a constituição de comitês interinstitucionais para a discussão dos processos tecnológicos em curso e dos desejados para a Educação na Saúde. 0 modelo do Campus Virtual de Saúde da OPAS ${ }^{49}$, sua organização em redee o protagonismo que algumas escolas assumem nesse processo também permitem exercitar a liderança e a construção coletiva mencionada. Fa- zer convergir experiências e conhecimento acumulados na aplicação das tecnologias de informação no campo das I nformações em Saúdecom as da Educação é mister para reunir atores e as pautas que se tangenciam. A proposição de um Ambiente Informacional para a Saúde, realizada por Vasconcellos ${ }^{11}$, ilustra:

[...] ampliar as potencialidades de uso das TI na gestão da Saúde implica, dentre outrasiniciativas, em uma nova concepção de organização das informações em saúde, onde sejam estruturados mecanismos e condições que criem um ambiente propício para o estabelecimento de uma sinergia de competências, recursos e memórias, frutos e matrizes do conhecimento sanitário, coletivamente produzido por seus sujeitos históricos.

Esse ambiente proposto por Vasconcellos ${ }^{11}$ tem como eixos orientadores a integração e articulação de informações em saúde ea convergência de tecnologias. A abordagem metodológica busca 0 aprofundamento da circulação inter/ transdiciplinar do conhecimento entre campos de saberes e propõe uso intensivo de tecnologias de informação que suscitem modos de cooperação ágeis etransversais, em um compartilhamento coordenado entre centros de decisão.

Destaca-se positivamentequeo SU Sérico em experiências colegiadas e em formatos democráticos para a composição e encaminhamento de agendas. A diversidade de atores como instituições deensino, redes deserviços, Secretarias Estaduais e Municipais, Ministério da Saúde, comissões, associações profissionais e Consel hos favorecem um debateheterogêneo edemocrático. Reunir atores-chaves para exercitar a definição conjunta de conteúdos essenciais aos processos educativos na saúde contribui para relações mais simétricas no mercado simbólico de produção cultural, no qual, segundo M artel eto ${ }^{59}$, os sistemas de ensino desempenham um papel instrumental na apropriação da riqueza simbólica queéjulgada digna de ser possuída e cultivada [...] e os bens culturais produzidos como matéria informacional não são compartilhados socialmente, e sim distribuídos, isto é, dependem de instâncias de produção, reprodução, transmi ssão e aquisição $0^{59}$.

Finalmente, a produção de objetos de aprendizagem, o exercício do consenso na padronização de metadados e o compartilhamento de conteúdos em rede (e, portanto, de conhecimento) trazem para a rede de escolas e instituições de ensino de saúde o desafio de estabelecer o que Levy ${ }^{60}$ chama de uma cooperação competitiva e uma competição cooperativa. Nesses jogos, os vencedores utilizam e aumentam a inteligência 
disponível e cooperaram de forma mais eficiente parauma inteligência coletiva; nestecaso, em benefício de uma mais abrangente e completa Política de Educação na Saúde para o SUS.

\section{Colaboradores}

M TL Cavalcante participou da concepção, análise e redação do artigo. M M Vasconcellos participou da concepção, análise e revisão crítica do artigo.

\section{Referências}

1. Brasil. M inistério da Saúde. Secretaria de Gestão do Trabalho e da Educação na Saúde. Informações Institucionais. [acessado $2006 \mathrm{M}$ ar 3]. Disponível em: http://portal.saude.gov.br/portal/sgtes/default.cfm

2. Brasil. M inistério da Saúde. Secretaria de Gestão do Trabalho e da Educação na Saúde. Departamento de Gestão da Educação na Saúde. Política de educação e desenvolvimento para o SUS: caminhos para a educação permanente em saúde: pólos de educação permanente em saúde. Brasília: M inistério da Saúde; 2004.

3. Brasil. M inistério da Saúde. Secretaria de Gestão do Trabalho e da Educação na Saúde. Documentos pre paratórios para 3a Conferência Nacional de Gestão do Trabalho e da Educação na Saúde. Trabalhadores da Saúde e a Saúde de Todos os Brasileiros: Práticas de Trabalho, Gestão, Formação e Participação. Brasília: Conselho Nacional de Saúde; 2005.

4. Struchiner $M$, et al. Novas tecnologias de informação e educação em saúde diante da revolução comunicacional e informacional. In: M inayo M CS, Coimbra Jr CEA, organizadores. Críticas e atuantes: $\mathrm{Ci}$ ências sociais e humanas em saúde na América Latina. Rio de Janeiro: Editora Fiocruz; 2005.

5. Santos JCT, Baungarten M. Sociedade da informação: as metodologias inovadoras no ensino contemporâneo da sociologia. In: Martin CB, organizador. Para onde vai a pós-graduação em Ciências Sociais no Brasil. Florianópolis: AN POCS/ EDUSC; 2005. 
6. Lévy P. As tecnologias da inteligência - o futuro do pensamento na era da informática. São Paulo: Editora 34; 1993.

7. Moraes IHS. Informação em saúde: da prática fragmentada ao exercício da cidadania. São Paulo e Rio de Janeiro: Hucitec/Abrasco; 1994.

8. M oraes I.H.S. Informações em saúde: para andarilhos e argonautas de uma tecnodemocracia emancipadora [tese de doutorado]. Rio de Janeiro (RJ): Escola Nacional de Saúde Pública, Fundação Oswaldo Cruz; 1998.

9. Iturri J. Ciberespaço e negociações de sentido: aspectos sociais da implementação de redes digitais de comunicação em instituições acadêmicas de saúde pública. Cad. Saúde Pública 1998; 14(4):803-810.

10. Leão BF. Padrões para representar a informação em saúde. In: Série Fiocruz, Eventos Científicos 3. Seminário Nacional de Informação e Saúde: 0 Setor de Saúde no Contexto da Sociedade da Informação. Rio de Janeiro: Fundação Oswaldo Cruz; 2000.

11. Vasconcellos M M, et al. Política de saúde e potencialidades de uso das tecnologias de informação. Saúde em Debate 2002; 61.

12. Misoczky MCA, et al. Educação à distância: reflexões críticas e experiências em saúde. Porto Alegre: Dacasa Editora; 2001.

13. Christante $L$, et al. $O$ papel do ensino à distância na educação médica continuada: uma análise crítica. Rev. Assoc. Med. Bras 2003; 49(3):326-329.

14. Costa KLD, et al. Utilizando a educação à distância na promoção da educação continuada em telemedicina. IX Congresso Brasileiro de Informática em Saúde. Ribeirão Preto; 2004.

15. Oliveira, MAN, Servo MLS.A educação à distância como estratégia da educação permanente do enfermeiro em centro cirúrgico frente as novas tecnologias. Sitientibus 2004; 30:9-20.

16. Tomaz JBC. Educação à distância como estratégia de capacitação permanenteem saúde: um relato deexperiência. Escola de Saúde Pública do Ceará. Congresso da Associação Brasileira de Educação a Distância. ABED 2004. [acessado 2006 Mai 4]. Disponível em: http:// www.abed.org.br/congresso2004/por/htm/169-TCD 4.htm

17. Belloni ML. Ensaio sobre a educação à distância no Brasil. Educação \& Sociedade 2002; 78: 117-142.

18. Jimenez RV. Educación, poder y mercado: deconstrucción crítica de los efectos disciplinantes de las TIC en la nueva Escuela del Espectáculo. Interface 2005; 9(18):475-488.

19. Nunes TCM. A especializaçäo em saúde pública e os serviços de saúde no Brasil de 1970 a 1989 [tese de doutorado]. Rio de Janeiro (RJ): Escola Nacional de Saúde Pública, Fundação Oswaldo Cruz; 1998.

20. Cury CRJ. Componentes básicos do fenômeno educativo. In: Cury CRJ. Educação e contradição. São Paulo: Cortez; 1995.

21. Yourdon E, Argila C. Análise e projeto orientados a objetos. São Paulo: Makron Books; 1999.

22. Ambler SW. Análise e projeto orientados a objetos: um guia para o desenvolvimento de aplicações orientadas a objetos. Rio de Janeiro: Infobook; 1997.

23. Tarouco LMR, et al. Reusabilidade de objetos educacionais. Revista N ovas Tecnologias na Educação 2003. [acessado 2005 Dez 12]. Disponível em: http:// www.cinted.ufrgs.br/renote
24. Jacobsen P. Reusable Learning Objects. What does the future holds? Learning and Training Innovations Newsline 2001. [acessado 2006 Jan 20]. Disponível em: http://www.Itimagazine.com/Itimagazine/article/articleD etail.jsp?id $=5043$

25. Polsani PR. Use and Abuse of Reusable Learning Objects. Journal of Digital Information 2003; 3:164. [acessado 2006 Fev 10]. Disponível em: http://jodi. ecs.soton.ac.uk/Articles/v03/i04/Polsani/

26. IEEE Learning Technology Standards Committee (LTSC) (2001). Draft Standard for Learning O bject M etadata Version 6.1. [acessado 2006 Fev 14]. Disponível em: http://ltsc.ieee.org/doc/

27. Wiley DA. Connecting learning objects to instructional design theory: a definition, a metaphor, and a taxonomy: the instructional use of learning objects. In: Agency for Instructional Technology 2002. [acessado 2005 Dez 2]. Disponível em: http://reusability.org/ $\mathrm{read} / \mathrm{chapters/willey.doc}$

28. L'Allier JJ. Frame of Reference: NETg's M ap to the Products, Their Structure and Core Beliefs. NetG.1997. [acessado 2006 Fev 14]. Disponível em: http://www.netg.com/research/whitepapers/ frameref.asp

29. Warpechowski M, Oliveira JPM. Obtenção de metadados de objetos de aprendizagem no AdaptWeb $\circledast$. Porto Alegre: Universidade Federal do Rio Grande do Sul; 2005. [acessado $2006 \mathrm{M}$ ar 10]. Disponível em: http:/ /www.inf.ufrgs.br/ erbd2005/Artigos/7924.pdf

30. Kraemer LLB. M etadados: estudo de sua aplicação no tratamento de recursos virtuais e análise de um projeto do programa prossiga do IBICT [tese de mestrado]. Curitiba (PR): Centro Federal de Educação Tecnológica do Paraná; 2001.

31. Biblioteca Regional de M edicina - Biblioteca Virtual em Saúde. Bireme BVS Informações Institucionais. [acessado $2006 \mathrm{Fev}$ 04]. Disponível em: http:// www.bireme.br

32. ISO. International Organization for Standartization. [acessado $2006 \mathrm{M}$ ar 20]. Disponível em: http// www.iso.org

33. Organização Pan Americana de Saúde. Organização Mundial de Saúde. O Prontuário eletrônico do paciente na assistência, informação e conhecimento médico. São Paulo: M assad, Marim e Azevedo N eto Editores; 2003.

34. ADL. Advanced Distributed Learning. Sharable Content Object Reference Model (SCORM \&) 2004 2nd Edition Overview, 2004. [acessado 2006 Fev 9]. Disponível em: http://www.adlnet.org

35. IM S Global Learning Consortium, Inc. [acessado 2006 Mar 4]. Disponível em: http://www. imsproject.org

36. AICC - The Aviation Industry Computer-Based Training Committee. [acessado $2006 \mathrm{M}$ ar 4]. Disponível em: http://www.aicc.org

37. Alliance of Remote Instructional Authoring and Distribution N etworks for Europe (ARIADNE). [acessado 2006 Fev 22]. Disponível em: http://www. ariadne-eu.org

38. The M edbiquitous Consortium - Enabling Collaboration for Healthcare Education. [acessado $2006 \mathrm{M}$ ar 22]. Disponível em: http://www.medbiq.org 
39. Smothers V. Healthcare Learning $\mathrm{O}$ bject M etadata. Specifications and description document. Version 0.1. Sep M edbiquitous Consortium. 2004. [acessado 2006 Mar 24]. Disponível em: http://www.medbiq.org

40. Webservices definition. W3C: The World Wide Web Consortium (W3C) [acessado $2006 \mathrm{Abr}$ 3]. Disponível em: http://www.w3c.org

41. Domínguez JAA. Entornos integrados de enseñanza virtual. In: Cebrián M, organizador. Enseñanza virtual para la innovación universitaria. Madrid: $N$ arcea S.A de Ediciones; 2003.

42. Pedreño A. La cooperacíon y las TIC para la mejora de la calidad en la universitad. In: Sangrà A, Snmamed MG, organizadores. La transformación de las universidades a través de las TIC: discursos y práticas. Barcelona: Editorial UOC; 2004.

43. Universidade de São Paulo. Escola do Futuro, Laboratório Didático Virtual. [acessado 2006 Abr 13]. Disponível em: http://www.futuro.usp.br

44. Baruque CB. Desenvolvimento de bibliotecas digitais de objetos de aprendizagem utilizando técnicas de datawarehousing e datamining [tese de doutorado]. Rio de Janeiro (RJ): Pontifícia Universidade Católica Rio de Janeiro; 2005.

45. M oura SL. U ma arquitetura para a integração de repositórios de objetos de aprendizagem baseada em mediadores e serviços Web [tese de mestrado]. Rio de Janeiro (RJ): Pontifícia Universidade Católica Rio de Janeiro; 2005.

46. Brauner DN. U ma arquitetura para catálogo de objetos baseada em ontologias [tese de mestrado]. Rio de Janeiro (RJ): Pontifícia Universidade Católica Rio de Janeiro; 2005.

47. Brasil. M inistério da Educação. Secretaria de Educação à Distância. RIVED - Rede Interativa Virtual de Educação. [acessado 2006 N ov 20]. Disponível em: http://ww2.relpe.org/relpe

48. Red Latinoamericana de Portales Educativos. [acessado 2006 Nov 20]. Disponível em: http://ww2. relpe.org/relpe

49. Organização Pan Americana de Saúde. Campus Virtual. [acessado 2006 Nov 10]. Disponível em: http:// www.campusvirtualsp.org
50. Santos M . Por uma outra globalização: do pensamento único à consciência universal. Rio de Janeiro: Record; 2001.

51. Latour B. Ciencia en acción. Barcelona: Editorial Labor; 1992.

52. Hanseth $O$, Aanestad M, Berg M . Actor-network theory and information systems. What's so special? Guest editors' introduction. Information Technology \& People 2004; 17(2):116-123.

53. Vink D. Itineraires. Praxeologiques en Sciences: Politique de la Science e de la Technologie, Du Laboratoire aux Reseaux. Le Travail Scientifique em M utation. Bruxelas: Comission das Communantes Europeeneres; 1992.

54. Maturana H. La realidad: objetiva o construída? Tomo II. Fundamentos biológicos del conocimiento. Barcelona: Anthropos; 1992.

55. Artmann E. Interdisciplinariedade no enfoque intersubjetivo habermasiano: reflexões sobre planejamento e AIDS. Rev C S Coletiva 2001; 6(1):183-195.

56. Santos Junior $S$, et al. Dificuldades para o uso das tecnologias da informação. RAE eletrônica 2005; 4(2). [acessado $2006 \mathrm{Fev}$ 14]. Disponível em: http:// www.scielo.br/pdf/raeel/v4n2/v4n2a05.pdf

57. Matus C. Política, planejamento e governo. Brasília: IPEA; 1993.

58. Flores F. Una crisis en el liderazgo. [acessado 2006 Mar 24]. Disponível em: http://www.atinachile.cl/ pdf/una crisis en_el_liderazgo.pdf

59. Marteleto RM . Cultura informacional: construindo o objeto informação pelo emprego dos conceitos de imaginário, instituição e campo social. Ciência da Informação 1995; 24(1).

60. Lévy P. Collective Intelligence. Development of Culture and Culture Development. Presentation for the Advisory Committee on Health Research of the Pan American Health Organization. Washington; 2002.

Artigo apresentado em 10/07/2006

Artigo aprovado em 14/11/2006

Versão final apresentada em 07/12/2006 